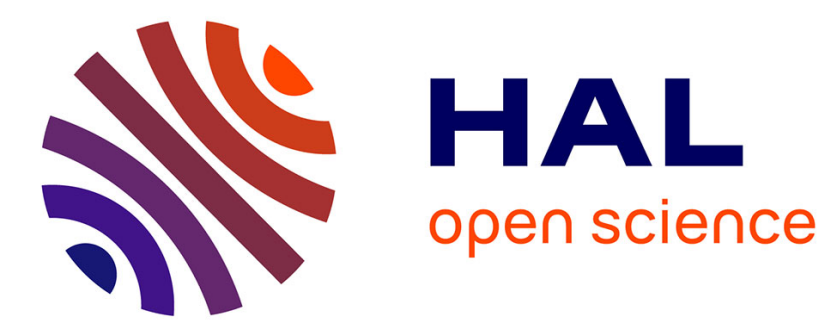

\title{
Uncertain Logical Gates in Possibilistic Networks. An Application to Human Geography
}

Didier Dubois, Giovanni Fusco, Henri Prade, Andrea G. B. Tettamanzi

\section{To cite this version:}

Didier Dubois, Giovanni Fusco, Henri Prade, Andrea G. B. Tettamanzi. Uncertain Logical Gates in Possibilistic Networks. An Application to Human Geography. Scalable Uncertainty Management, Sep 2015, Québec, QC, Canada. pp.249-263, 10.1007/978-3-319-23540-0_17 . hal-01203515

\section{HAL Id: hal-01203515 https://inria.hal.science/hal-01203515}

Submitted on 23 Sep 2015

HAL is a multi-disciplinary open access archive for the deposit and dissemination of scientific research documents, whether they are published or not. The documents may come from teaching and research institutions in France or abroad, or from public or private research centers.
L'archive ouverte pluridisciplinaire HAL, est destinée au dépôt et à la diffusion de documents scientifiques de niveau recherche, publiés ou non, émanant des établissements d'enseignement et de recherche français ou étrangers, des laboratoires publics ou privés. 


\title{
Uncertain Logical Gates in Possibilistic Networks. An Application to Human Geography
}

\author{
Didier Dubois ${ }^{1}$, Giovanni Fusco ${ }^{2}$, Henri Prade ${ }^{1}$, and Andrea Tettamanzi ${ }^{3}$ \\ 1 IRIT - CNRS, 118, route de Narbonne, Toulouse, France \\ 2 Univ. Nice Sophia Antipolis/CNRS, ESPACE, UMR7300, Nice, France \\ 3 Univ. Nice Sophia Antipolis/CNRS, I3S, UMR7271, Sophia Antipolis, France \\ duboisdirit.fr, fuscodunice.fr, pradedirit.fr, \\ andrea.tettamanzi@unice.fr
}

\begin{abstract}
Possibilistic networks offer a qualitative approach for modeling epistemic uncertainty. Their practical implementation requires the specification of conditional possibility tables, as in the case of Bayesian networks for probabilities. This paper presents the possibilistic counterparts of the noisy probabilistic connectives (and, or, max, min, ....). Their interest is illustrated on an example taken from a human geography modeling problem. The difference of behaviors in some cases of some possibilistic connectives, with respect to their probabilistic analogs, is discussed in details.
\end{abstract}

\section{Introduction}

Bayesian networks [11] can be built in two ways: statistical and subjective. In the first case, a supposedly large dataset involving a number of variables is available, and the Bayesian network is obtained by some machine learning procedure. The probability tables thus obtained have a frequentist flavor, and the simplest network possible is searched for. On the contrary, Bayesian networks can be specified using expert knowledge. In this case, the structure of a network relating the variables is first given, often relying on causal connections between variables and conditional independence relations the expert is aware of. Then probability tables must be filled by the expert. They consist, for each variable in the network, of conditional probabilities for that variable, conditioned on each configuration of its parent variables. Note that, even if causal relations as perceived by the expert are instrumental in building a simple and interpretable network, the joint probability distribution obtained by combining the probability tables no longer accounts for causality. Another difficulty arises for causality-based Bayes networks: if variables are not binary and/or the number of parent variables is more than two, the task of eliciting numerical probability tables becomes tedious, if not impossible to fulfill. Indeed, the number of probability values to be supplied increases exponentially with the number of parent variables.

To alleviate the elicitation task, the notion of noisy logical gate (or connective) has been introduced, based on the assumption of independent causal influences that can be combined. As a result, one small conditional probability table is elicited per parent variable, and the probability table of each variable given its parents is obtained by combining these small tables by a so-called noisy connective $[6,10]$, which may include a 
so-called leakage factor summarizing the causal effect of variables not explicitly present in the network.

While the notion of noisy connective solves the combinatorial problem of collecting many probability values to a large extent, the issue remains that people cannot always provide precise probability assessments. Let alone the fact that the probability scale is too fine-grained for human perception of belief or frequencies, some conditional probability values may be ill-known or plainly unknown to the experts. The usual Bayesian recommendation in the latter case is to use uniform distributions, but it is well-known that these do not properly model ignorance. Alternatively, one may use imprecise probability networks (called credal networks) [12], qualitative Bayesian networks [14] or possibilistic networks [3]. While the two first options extend probabilistic networks to ill-known parameters (with an interval-based approach for the former and an ordinal approach for the latter), possibilistic networks represent a more drastic departure from probabilistic networks. In their qualitative version, possibilistic networks can be defined on a finite chain of possibility values and do not refer to numerical values. This feature may make the collection of expert information on conditional tables easier than requiring precise numbers obeying the laws of probability.

In this paper, we propose possibilistic counterparts of noisy connectives of probabilistic networks. As possibilistic uncertainty is merely epistemic and due to a lack of information, we shall speak of uncertain connectives. After recalling probabilistic networks with noisy gates, we present the corresponding approach for possibilistic networks and present various uncertain gates, especially the AND, OR, MAX, and MIN functions. ${ }^{4}$ Finally, the approach, including algorithmic issues, is illustrated on a belief network stemming from an application to human geography.

\section{Probabilistic Networks with Independent Causal Influences}

Consider a set of independent variables $X_{1}, \ldots, X_{n}$ that influence the value of a variable $Y$. In the ideal case, there is a deterministic function $f$ such that $Y=f\left(X_{1}, X_{2}, \ldots, X_{n}\right)$. In order to account for uncertainty, one may assume the existence of intermediary variables $Z_{1}, \ldots, Z_{n}$, such that $Z_{i}$ expresses the fact that $X_{i}$ will have a causal influence on $Y$, and to what extent $\left(Z_{i}\right.$ has the same domain as $Y$ ). It is assumed that the relation between $X_{i}$ and $Z_{i}$ is probabilistic and that $X_{i}$ is independent of other variables given $Z_{i}$. Besides, we consider the deterministic function as affected by the auxiliary variables $Z_{i}$ only. In other words, we get a probabilistic network such that

$$
P\left(Y, Z_{1}, \ldots, Z_{n}, X_{1}, X_{2}, \ldots, X_{n}\right)=P\left(Y, Z_{1}, \ldots, Z_{n}\right) \cdot \prod_{i=1}^{n} P\left(Z_{i} \mid X_{i}\right)
$$

\footnotetext{
${ }^{4}$ The idea of possibilistic uncertain gates was first considered empirically by [13] directly in the setting of possibilistic logic, at a time where possibilistic networks had not yet been introduced. It seems that the question of possibilistic uncertain gates has not been reconsidered ever since, if we except a recent study in the broader setting of imprecise probabilities [1] and a preliminary outline in French by the authors [4].
} 
where $P\left(Y, Z_{1}, \ldots, Z_{n}\right)=1$ if $Y=f\left(Z_{1}, Z_{2}, \ldots, Z_{n}\right)$ and 0 otherwise. This is called a noisy function. In particular, notice that the dependence tables between $Y$ and $X_{1}, \ldots, X_{n}$ can now be obtained by combining simple conditional probability distributions pertaining to single factors:

$$
P\left(y \mid x_{1}, \ldots, x_{n}\right)=\sum_{z_{1}, \ldots, z_{n}: y=f\left(z_{1}, \ldots, z_{n}\right)} \prod_{i=1}^{n} P\left(z_{i} \mid x_{i}\right) .
$$

This is the assumption of independence of causal influence (ICI) [6]. In the case of Boolean variables, it is assumed that $P\left(z_{i}=0 \mid x_{i}=0\right)=1$ (no cause, no effect), while $P\left(z_{i}=0 \mid x_{i}=1\right)$ can be positive (the effect may or may not appear when the cause is present).

Canonical ICI models are obtained by means of specific choice of the function $f$. For instance, if all variables are Boolean, $f$ will be a logical connective. In this case, we speak of noisy OR $(f=\vee)$, noisy AND $(f=\wedge)$; if the range of the $Z_{i}$ 's and $Y$ is a totally ordered set, usual gates are the noisy $\operatorname{MAX}(f=\max )$, or MIN $(f=\min )$.

The approach may be further refined by allowing $f$ to summarize the potential effect of external variables not taken into account: this is the leaky model. Now, $Y$ also depends on a leak variable $Z_{\ell}$ not explicitly related to specifically identified causes, i.e., $Y=f\left(Z_{1}, Z_{2}, \ldots, Z_{n}, Z_{\ell}\right)$. The domain of $Z_{\ell}$ is supposed to be the range of $f$, i.e., the domain of $Y$ and this variable is independent of the other ones. Hence, the leakage model may be written as:

$$
P\left(Y, Z_{1}, \ldots, Z_{n}, Z_{\ell}, X_{1}, \ldots, X_{n}\right)=P\left(Y, Z_{1}, \ldots, Z_{n}\right) \cdot P\left(Z_{\ell}\right) \cdot \prod_{i=1}^{n} P\left(Z_{i} \mid X_{i}\right)
$$

so that

$$
P\left(y \mid x_{1}, \ldots, x_{n}\right)=\sum_{z_{1}, \ldots, z_{n}, z_{\ell}: y=f\left(z_{1}, \ldots, z_{n}, z_{\ell}\right)} P\left(z_{\ell}\right) \cdot \prod_{i=1}^{n} P\left(z_{i} \mid x_{i}\right)
$$

For instance, in the case of Boolean variables, $P\left(y=1 \mid x_{1}=0, \ldots, x_{n}=0\right)$ may be positive due to such external causes.

We will now turn to the question whether the same kind of ICI approach can be used to elicit possibilistic networks as well.

\section{Canonical Possibilistic Networks}

Possibility theory $[7,16]$ is based on maxitive set functions associated to possibility distributions. Formally, given a universe of discourse $U$, a possibility distribution $\pi: U \rightarrow[0,1]$ pertains to a variable $X$ ranging on $U$ and represents the available (incomplete) information about the more or less possible values of $X$, assumed to be single-valued. Thus, $\pi(u)=0$ means that $X=u$ is impossible. The consistency of information is expressed by the normalization of $\pi: \exists u \in U, \pi(u)=1$, namely, at least one value is fully possible for $X$. Distinct values $u$ and $u^{\prime}$ may be simultaneously 
possible at degree 1. A state of complete ignorance is represented by the distribution $\pi_{?}(u)=1, \forall u \in U$. A possibility measure of an event $A \subseteq U$ is defined by

$$
\Pi(A)=\sup _{u \in A} \pi(u) .
$$

Possibility measures are maxitive, i.e.,

$$
\forall A, \forall B, \Pi(A \cup B)=\max (\Pi(A), \Pi(B)) .
$$

The underlying assumption is that the agent focuses on most plausible values, neglecting other ones. A dual measure of necessity $N(A)=1-\Pi(U \backslash A)$ expresses the certainty of event $A$ as the impossibility of non- $A$.

A possibilistic network $[2,3]$ has the same structure as a Bayesian network. The joint possibility for $n$ variables linked by an acyclic directed graph is defined by

$$
\pi\left(x_{1}, \ldots, x_{n}\right)=*_{i=1, \ldots, n} \pi\left(x_{i} \mid p a\left(X_{i}\right)\right),
$$

where $x_{i}$ is an instantiation of the variable $X_{i}$, and $p a\left(X_{i}\right)$ an instantiation of the parent variables of $X_{i}$. The operation $*$ is the minimum (in the qualitative case) or the product (in the numerical case).

Deterministic models $Y=f\left(X_{1}, \ldots, X_{n}\right)$ are defined as in the probabilistic case:

$$
\pi\left(y \mid x_{1}, \ldots, x_{n}\right)= \begin{cases}1 & \text { if } y=f\left(x_{1}, \ldots x_{n}\right) \\ 0 & \text { otherwise }\end{cases}
$$

Let us define possibilistic models with independent causal influences (ICI). We use a deterministic function $Y=f\left(Z_{1}, \ldots, Z_{n}\right)$ with $n$ intermediary causal variables $Z_{i}$, as for the probabilistic models. Now, $\pi\left(y \mid x_{1}, \ldots, x_{n}\right)$ is of the form:

$$
\pi\left(y \mid z_{1}, \ldots, z_{n}\right) * \pi\left(z_{1}, \ldots, z_{n} \mid x_{1}, \ldots, x_{n}\right),
$$

where $\pi\left(y \mid z_{1}, \ldots, z_{n}\right)$ obeys Equation 4. Again, each variable $Z_{i}$ only depends (in an uncertain way) on the variable $X_{i}$. Thus, we have $\pi\left(z_{1}, \ldots, z_{n} \mid x_{1}, \ldots, x_{n}\right)=$ $*_{i=1, \ldots, n} \pi\left(z_{i} \mid x_{i}\right)$. This leads to the equality

$$
\pi\left(y \mid x_{1}, \ldots, x_{n}\right)=\max _{z_{1}, \ldots, z_{n}: y=f\left(z_{1}, \ldots, z_{n}\right)} *_{i=1, \ldots, n} \pi\left(z_{i} \mid x_{i}\right),
$$

whose similarity with Eq. 2 is striking. Notice that, when $*=$ min, Eq. 5 boils down to applying the extension principle [16] to function $f$, assuming fuzzy-valued inputs $F_{1}, \ldots, F_{n}$, where the membership function of $F_{i}$ is defined by $\mu_{F_{i}}\left(z_{i}\right)=\pi\left(z_{i} \mid x_{i}\right)$.

In case we suppose that $y$ depends also in an uncertain way on other causes summarized by a leak variable $Z_{\ell}$, then the counterpart of Eq. 3 reads:

$$
\pi\left(y \mid x_{1}, \ldots, x_{n}\right)=\max _{z_{1}, \ldots, z_{n}, z_{\ell}: y=f\left(z_{1}, \ldots, z_{n}, z_{\ell}\right)} *_{i=1, \ldots, n} \pi\left(z_{i} \mid x_{i}\right) * \pi\left(z_{\ell}\right) .
$$

In the following, we provide a detailed analysis of possibilistic counterparts of noisy gates. 


\subsection{Uncertain OR and AND Gates}

The variables are assumed to be Boolean (i.e., $Y=y$ or $\neg y$, etc.). The uncertain OR (counterpart of the probabilistic "noisy OR") assumes that $X_{i}=x_{i}$ for at least one variable $X_{i}$ represents a sufficient cause for getting $Y=y$, and $Z_{i}=z_{i}$ indicates that $X_{i}=x_{i}$ has caused $Y=y$. This gives $f\left(Z_{1}, \ldots, Z_{n}\right)=\bigvee_{i=1}^{n} Z_{i}$. The uncertainty indicates that the causes may fail to produce their effects. $Z_{i}=\neg z_{i}$ indicates that $X_{i}=x_{i}$ did not cause $Y=y$ due to the presence of some inhibitor that prevents the effect from taking place. We assume it is more possible that $X_{i}=x_{i}$ causes $Y=y$ than the opposite (otherwise one could not say that $X_{i}=x_{i}$ is sufficient for causing $Y=y)$. Then we must define $\pi\left(z_{i} \mid x_{i}\right)=1$ and $\pi\left(\neg z_{i} \mid x_{i}\right)=\kappa_{i}<1$. Besides, $\pi\left(z_{i} \mid \neg x_{i}\right)=0$, since when $X_{i}$ is absent, it does not cause $y$. Hence the causal elementary possibility table:

$$
\begin{array}{c|c|c}
\pi\left(Z_{i} \mid X_{i}\right) & x_{i} & \neg x_{i} \\
\hline z_{i} & 1 & 0 \\
\hline \neg z_{i} & \kappa_{i} & 1
\end{array}
$$

Note that in the case of a probabilistic network, $\pi\left(z_{i} \mid x_{i}\right)$ is replaced by $1-\kappa_{i}$ in the above table. We can then obtain the table of the conditional possibility distribution $\pi\left(Y \mid X_{1}, \ldots, X_{n}\right)$ by means of Eq. 5 :

$$
\begin{aligned}
& \pi\left(y \mid X_{1}, \ldots, X_{n}\right)=\max _{z_{1}, \ldots, z_{n}: z_{1} \vee \cdots \vee z_{n}=1} *_{i=1}^{n} \pi\left(z_{i} \mid X_{i}\right) \\
& =\max _{i=1}^{n} \pi\left(z_{i} \mid X_{i}\right) *\left(*_{j \neq i} \max \left(\pi\left(z_{j} \mid X_{j}\right) \pi\left(\neg z_{j} \mid X_{j}\right)\right) ;\right. \\
& \pi\left(\neg y \mid X_{1}, \ldots, X_{n}\right)=\max _{z_{1}, \ldots, z_{n}: z_{1} \vee \cdots \vee z_{n}=0} *_{i=1}^{n} \pi\left(z_{i} \mid X_{i}\right) \\
& =\pi\left(\neg z_{1} \mid X_{1}\right) * \cdots * \pi\left(\neg z_{n} \mid X_{n}\right) .
\end{aligned}
$$

Let us denote by $\mathbf{x}$ a configuration of $\left(X_{1}, \ldots, X_{n}\right)$, and let $I_{+}(\mathbf{x})=\left\{i: X_{i}=x_{i}\right\}$ and $I_{-}(\mathbf{x})=\left\{i: X_{i}=\neg x_{i}\right\}$. Then we get:

- $\pi(\neg y \mid \mathbf{x})=*_{i=1, \ldots, n} \pi\left(\neg z_{i} \mid X_{i}=\mathbf{x}_{i}\right)=*_{i \in I_{+}(\mathbf{x})} \kappa_{i} ;$

- $\pi(y \mid \mathbf{x})=1$ when $\mathbf{x} \neq\left(\neg x_{1}, \ldots, \neg x_{n}\right)$;

- $\pi\left(\neg y \mid \neg x_{1}, \ldots, \neg x_{n}\right)=1, \pi\left(y \mid \neg x_{1}, \ldots, \neg x_{n}\right)=0: \neg y$ (no effect) can be obtained for sure only if all the causes are absent.

For $n=2$, this gives the conditional tables:

$$
\begin{array}{c|c|c}
\pi\left(y \mid X_{1} X_{2}\right) & x_{1} & \neg x_{1} \\
\hline x_{2} & 1 & 1 \\
\hline \neg x_{2} & 1 & 0
\end{array} \quad \begin{array}{cc|c|c}
\pi\left(\neg y \mid X_{1} X_{2}\right) & x_{1} & \neg x_{1} \\
\hline x_{2} & \kappa_{1} * \kappa_{2} & \kappa_{2} \\
\hline \neg x_{2} & \kappa_{1} & 1
\end{array}
$$

More generally, if there are $n$ causes, we have to provide the values of $n$ parameters $\kappa_{i}$.

For the uncertain OR with leak, we now assume that $f\left(Z_{1}, \ldots, Z_{n}\right)=\bigvee_{i=1}^{n} Z_{i} \vee$ $Z_{\ell}$, where $Z_{\ell}$ is an unknown external cause. We assign $\pi\left(z_{\ell}\right)=\kappa_{\ell}<1$ considering that $z_{\ell}$ is not a usual cause. We thus obtain

- $\pi(\neg y \mid \mathbf{x})=*_{i=1, \ldots, n} \pi\left(\neg z_{i} \mid X_{i}=\mathbf{x}_{i}\right) * \pi\left(\neg z_{\ell}\right)=*_{i \in I_{+}(\mathbf{x})} \kappa_{i} ;$

- $\pi(y \mid \mathbf{x})=1$, if $\mathbf{x} \neq\left(\neg x_{1}, \ldots, \neg x_{n}\right)$;

$-\pi\left(\neg y \mid \neg x_{1}, \ldots, \neg x_{n}\right)=1$; 
- $\pi\left(y \mid \neg x_{1}, \ldots, \neg x_{n}\right)=\kappa_{\ell}$ (even if the causes $x_{i}$ are absent, there is still a possibility for having $Y=y$, namely if the external cause is present).

Indeed, we get (letting $\neg x=\neg x_{1}, \ldots, \neg x_{n}$ ),

$$
\begin{aligned}
\pi\left(y \mid \neg x_{1}, \ldots, \neg x_{n}\right) & \left.=\max \left(\pi\left(y \mid \neg x, z_{\ell}\right) * \pi\left(z_{\ell}\right), \pi\left(y \mid \neg x, \neg z_{\ell}\right) * \pi\left(\neg z_{\ell}\right)\right)\right) \\
& =\max \left(1 * \kappa_{\ell}, 0 * 1\right)=\kappa_{\ell} .
\end{aligned}
$$

For $n=2$, the conditional table becomes:

$$
\begin{array}{c|c|cc|c|c}
\pi\left(y \mid X_{1} X_{2}\right) & x_{1} & \neg x_{1} \\
\hline x_{2} & 1 & 1 \\
\hline \neg x_{2} & 1 & \kappa_{\ell}
\end{array} \quad \begin{array}{cc|c|c|}
\pi\left(\neg y \mid X_{1} X_{2}\right) & x_{1} & \neg x_{1} \\
\hline x_{2} & \kappa_{1} * \kappa_{2} & \kappa_{2} \\
\hline \neg x_{2} & \kappa_{1} & 1
\end{array}
$$

The only 0 entry has been replaced by the leakage coefficient. For $n$ causes, we have now to provide the values of $n+1$ parameters $\kappa_{i}$.

The uncertain AND (counterpart of the probabilistic "noisy AND") uses the same local conditional tables but it assumes that $X_{i}=x_{i}$ represents a necessary cause for $Y=y$. We again build the conditional possibility table $\pi\left(Y \mid X_{1}, \ldots, X_{n}\right)$ by means of Eq. 5 with $f\left(Z_{1}, \ldots, Z_{n}\right)=\bigwedge_{i=1}^{n} Z_{i}$. Thus, we find

- $\pi\left(\neg y \mid x_{1}, \ldots, x_{n}\right)=\max _{z_{1}, \ldots, z_{n}: \neg y=z_{1} \wedge \cdots \wedge z_{n}} *_{i=1}^{n} \pi\left(z_{i} \mid x_{i}\right)=\max _{i=1}^{n} \pi\left(\neg z_{i} \mid\right.$ $\left.x_{i}\right)=\max _{i=1}^{n} \kappa_{i}$;

- $\pi\left(y \mid x_{1}, \ldots, x_{n}\right)=1$

$-\pi(\neg y \mid \mathbf{x})=1, \pi(y \mid \mathbf{x})=0$ if $\mathbf{x} \neq\left(x_{1}, \ldots, x_{n}\right)$ (if at least one of the causes is absent, the effect is necessarily absent).

For $n=2$, Eq. 5 yields the conditional tables:

$$
\begin{array}{c|c|cc|c|c}
\pi\left(y \mid X_{1} X_{2}\right) & x_{1} & \neg x_{1} \\
\hline x_{2} & 1 & 0 \\
\hline \neg x_{2} & 0 & 0
\end{array} \quad \begin{array}{ccc}
\pi\left(\neg y \mid X_{1} X_{2}\right) & x_{1} & \neg x_{1} \\
\hline x_{2} & \max \left(\kappa_{1}, \kappa_{2}\right) & 1 \\
\hline \neg x_{2} & 1 & 1
\end{array}
$$

More generally, if there are $n$ causes, we have to assess $n$ values for the parameters $\kappa_{i}$. The case of the uncertain AND with leak corresponds to the possibility $\pi\left(z_{L}\right)=$ $\kappa_{L}<1$ that an external factor $Z_{L}=z_{L}$ causes $Y=y$ independently of the values of the $X_{i}$. Namely $f\left(Z_{1}, \ldots, Z_{n}, Z_{L}\right)=\left(\bigwedge_{i=1}^{n} Z_{i}\right) \vee Z_{L}$. For $n=2$, Eq. 5 then gives the conditional tables:

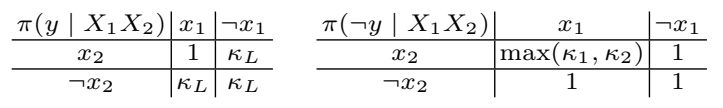

\subsection{Comparison with Probabilistic Gates}

It is interesting to compare the possibilistic and probabilistic tables. Consider those of the noisy OR [6], where $\kappa_{i}=P\left(\neg z_{i} \mid x_{i}\right)$ :

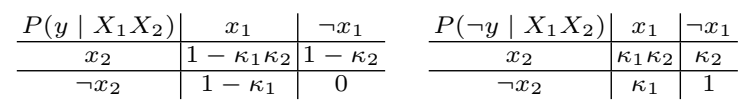


There is an important difference between the behaviors of uncertain and noisy OR if $*=$ min. In the possibilistic tables, we see (using the associated necessity measure $N$, and Boolean notations for the instantiations of $x_{1}$ and $\left.x_{2}\right)$ that $N(y \mid 11)=\max (N(y \mid$ $10), N(y \mid 01))$ while $P(y \mid 11)>\max (P(y \mid 10), P(y \mid 01))$, so that the presence of two causes does not reinforce the certainty of the effect wrt the presence of the most influential cause. Hence qualitative possibility networks will be less expressive than probabilistic networks. If $*=$ product, $N(y \mid 11)=1-\kappa_{1} \kappa_{2}>\max (N(y \mid$ $10), N(y \mid 01))$ as with the probability case.

Another major difference will occur in case the effects of causes are not frequent, as when $P\left(\neg z_{i} \mid x_{i}\right)=\kappa_{i}>0.5, i=1,2$. Then it may happen that $P\left(y \mid x_{1} x_{2}\right)=$ $1-\kappa_{1} \kappa_{2}>0.5$, that is the presence of the two causes makes the effect frequent. Then a possibilistic rendering of this case must be such that $\pi\left(\neg z_{i} \mid x_{i}\right)=1>\pi\left(z_{i} \mid x_{i}\right)=\lambda_{i}$ (say). However, there is no way of observing this reversal effect, since $\pi\left(y \mid x_{1} x_{2}\right)=$ $\max \left(\lambda_{1} * \lambda_{2}, \lambda_{1}, \lambda_{2}\right)=\max \left(\lambda_{1}, \lambda_{2}\right)<1$. Hence $\pi\left(\neg y \mid x_{1} x_{2}\right)=1$ and $N(y \mid$ $\left.x_{1} x_{2}\right)=0$. In other words, using the uncertain OR, two causes that are individually insufficient to make an effect plausible are still insufficient to make it plausible if joined together. Note that this fact reminds of the property of closure under conjunction for necessity measures in possibility theory $\left(N\left(y_{1}\right)>0\right.$ and $N\left(y_{2}\right)>0$ imply $N\left(y_{1} \wedge\right.$ $\left.y_{2}\right)>0$ ) which fail to hold in probability theory.

One way to address this problem is to define the global conditional possibility tables $\pi\left(Y \mid X_{1}, X_{2}\right)$ enforcing $\pi\left(y \mid x_{1} x_{2}\right)>\pi\left(\neg y \mid x_{1} x_{2}\right)$ even if $\pi\left(y \mid x_{1}\right)<\pi\left(\neg y \mid x_{1}\right)$ and $\pi\left(y \mid x_{2}\right)<\pi\left(\neg y \mid x_{2}\right)$, which is perfectly compatible with possibility theory. We will outline a solution of this kind in the next section for the uncertain MAX, which is a generalization of the uncertain OR. However, one cannot build the global table from the marginal ones using an uncertain OR.

\subsection{Uncertain MAX and MIN Gates}

The uncertain MAX is a multiple-valued extension of the uncertain OR, where the output variable (hence the variables $Z_{i}$ ) is valued on a finite, totally ordered, severity or intensity scale $L=\{0<1<\cdots<m\}$. We assume that $Y=\max \left(Z_{1}, \ldots, Z_{n}\right)$. $Z_{i}=z_{i} \in L$ represents the fact that $X_{i}$ alone has increased the value of $Y$ at level $z_{i}$. The conditional possibility distributions $\pi\left(y \mid x_{i}\right)$ are supposed to be given. We can then compute the conditional tables, as

$$
\begin{aligned}
\pi\left(y \mid x_{1}, \ldots, x_{n}\right) & =\max _{z_{1}, \ldots, z_{n}: y=\max \left(z_{1}, \ldots, z_{n}\right)} *_{i=1}^{n} \pi\left(z_{i} \mid x_{i}\right) \\
& =\max _{i=1}^{n} \pi\left(Z_{i}=y \mid x_{i}\right) *\left(*_{j \neq i} \Pi\left(Z_{j} \leq y \mid x_{j}\right)\right) .
\end{aligned}
$$

In a causal setting, we assume that $y=0$ is a normal state, and $y>0$ is more or less abnormal, $y=m$ being fully abnormal. Suppose that the domain of $X_{i}$ is $L$ as well. It is natural to assume that:

- if $X_{i}=j$ then $Z_{i}=j$, which means $\Pi\left(Z_{i}=j \mid X_{i}=j\right)=1$;

- $\Pi\left(Z_{i}>j \mid X_{i}=j\right)=0$ (a cause having a weak intensity cannot induce an effect with strong severity); 
- $0<\Pi\left(Z_{i}<j \mid X_{i}=j\right)<1$ (a cause having strong intensity may sometimes only induce an effect with weak severity, or may even have no effect at all);

- An effect with severity weaker than the intensity of a cause is all the less plausible as the effect is weak. This leads to suppose the following inequalities:

$0<\pi\left(Z_{i}=0 \mid X_{i}=j\right)<\pi\left(Z_{i}=1 \mid X_{i}=j\right)<\cdots<\pi\left(Z_{i}=j \mid X_{i}=j\right)=1$.

This leads to state the left-hand side table below (for 3 levels of strength $0,1,2$ ).

\begin{tabular}{c|c|c|c}
$\pi\left(Z_{i} \mid X_{i}\right)$ & $X_{i}=2$ & $X_{i}=1$ & $X_{i}=0$ \\
\hline$Z_{i}=2$ & 1 & 0 & 0 \\
\hline$Z_{i}=1$ & $\kappa_{i}^{12}$ & 1 & 0 \\
\hline$Z_{i}=0$ & $\kappa_{i}^{02}$ & $\kappa_{i}^{01}$ & 1
\end{tabular}

\begin{tabular}{c|c|c}
$\pi\left(Z_{i} \mid X_{i}\right)$ & $X_{i}=2$ & $X_{i}=0$ \\
\hline$Z_{i}=2$ & 1 & 0 \\
\hline$Z_{i}=1$ & $\kappa_{i}^{12}$ & 0 \\
\hline$Z_{i}=0$ & $\kappa_{i}^{02}$ & 1
\end{tabular}

where $0<\kappa_{i}^{02}<\kappa_{i}^{12}<1,0<\kappa_{i}^{01}<1$. In case we have $m$ levels of strength, we have to assess $\frac{m(m+1)}{2}$ coefficients. On the right-hand side is the corresponding table when the variables $X_{i}$ are Boolean (then the middle column is dropped).

The global conditional possibility tables are thus obtained by applying Eq. 5, using the values of $\pi\left(Z_{i} \mid X_{i}\right)$, as given in the above table.

$$
\pi(Y=j \mid \mathbf{x})=\max _{i=1}^{n} \pi\left(Z_{i}=j \mid x_{i}\right) *\left(*_{\ell \neq i} \Pi\left(Z_{\ell} \leq j \mid x_{\ell}\right)\right) .
$$

For $n=2, m=2$, when the $X_{i}$ 's are three-valued and Boolean, respectively, the following conditional tables are obtained (in the Boolean case, only 4 lines remain):

\begin{tabular}{c|c|c|c}
$\mathbf{x}$ & $\pi(2 \mid \mathbf{x})$ & $\pi(1 \mid \mathbf{x})$ & $\pi(0 \mid \mathbf{x})$ \\
\hline$(2,2)$ & 1 & $\max \left(\kappa_{1}^{12}, \kappa_{2}^{12}\right)$ & $\kappa_{1}^{02} * \kappa_{2}^{02}$ \\
\hline$(2,1)$ & 1 & 1 & $\kappa_{1}^{02} * \kappa_{2}^{01}$ \\
\hline$(2,0)$ & 1 & $\kappa_{1}^{12}$ & $\kappa_{1}^{02}$ \\
\hline$(1,2)$ & 1 & 1 & $\kappa_{1}^{01} * \kappa_{2}^{02}$ \\
\hline$(1,1)$ & 0 & 1 & $\kappa_{1}^{01} * \kappa_{2}^{01}$ \\
\hline$(1,0)$ & 0 & 1 & $\kappa_{1}^{01}$ \\
\hline$(0,2)$ & 1 & $\kappa_{2}^{12}$ & $\kappa_{2}^{02}$ \\
\hline$(0,1)$ & 0 & 1 & $\kappa_{2}^{01}$ \\
\hline$(0,0)$ & 0 & 0 & 1
\end{tabular}

\begin{tabular}{c|c|c|c}
$\mathbf{x}$ & $\pi(2 \mid \mathbf{x})$ & $\pi(1 \mid \mathbf{x})$ & $\pi(0 \mid \mathbf{x})$ \\
\hline$(2,2)$ & 1 & $\max \left(\kappa_{1}^{12}, \kappa_{2}^{12}\right)$ & $\kappa_{1}^{02} * \kappa_{2}^{02}$ \\
\hline$(2,0)$ & 1 & $\kappa_{1}^{12}$ & $\kappa_{1}^{02}$ \\
\hline$(0,2)$ & 1 & $\kappa_{2}^{12}$ & $\kappa_{2}^{02}$ \\
\hline$(0,0)$ & 0 & 0 & 1
\end{tabular}

More generally, If we have $m$ levels of strength, and $n$ causal variables, we need $\frac{n m(m+1)}{2}$ coefficients for defining the uncertain MAX. If we take into account the leak, we have to add $\frac{m(m+1)}{2}$ coefficients per variable, in order to replace the 0 by a leak coefficient in the conditional tables $\pi\left(Z_{i} \mid X_{i}\right)$ (assuming that an effect of strong severity may take place even if the causes present have a weak intensity).

As for the uncertain MAX wrt uncertain OR, the uncertain MIN is a multiple-valued extension of the uncertain AND, where variables are valued on a the intensity scale $L=\{0<1<\cdots<m\}$. We assume that $Y=\min \left(Z_{1}, \ldots, Z_{n}\right)$. We can then compute the conditional tables, as

$$
\begin{aligned}
\pi\left(y \mid x_{1}, \ldots, x_{n}\right) & =\max _{z_{1}, \ldots, z_{n}: y=\min \left(z_{1}, \ldots, z_{n}\right)} *_{i=1}^{n} \pi\left(z_{i} \mid x_{i}\right) \\
& =\max _{i=1}^{n} \pi\left(Z_{i}=y \mid x_{i}\right) *\left(*_{j \neq i} \Pi\left(Z_{j} \geq y \mid x_{j}\right)\right) .
\end{aligned}
$$


The conditional possibility tables are thus obtained by applying Eq. 5, using the same values of $\pi\left(Z_{i} \mid X_{i}\right)$, as in the case of the uncertain MAX. For $n=2, m=2$, this gives the following conditional tables (for ternary and binary inputs, respectively):

\begin{tabular}{c|c|c|c}
$\mathbf{x}$ & $\pi(2 \mid \mathbf{x})$ & $\pi(1 \mid \mathbf{x})$ & $\pi(0 \mid \mathbf{x})$ \\
\hline$(2,2)$ & 1 & $\max \left(\kappa_{1}^{12}, \kappa_{2}^{12}\right)$ & $\max \left(\kappa_{1}^{02}, \kappa_{2}^{02}\right)$ \\
\hline$(2,1)$ & 0 & 1 & $\max \left(\kappa_{1}^{02}, \kappa_{2}^{01}\right)$ \\
\hline$(2,0)$ & 0 & $\kappa_{1}^{12}$ & 1 \\
\hline$(1,2)$ & 0 & 1 & $\max \left(\kappa_{1}^{01}, \kappa_{2}^{02}\right)$ \\
\hline$(1,1)$ & 0 & 1 & $\max \left(\kappa_{1}^{01}, \kappa_{2}^{01}\right)$ \\
\hline$(1,0)$ & 0 & 0 & 1 \\
\hline$(0,2)$ & 0 & $\kappa_{2}^{12}$ & 1 \\
\hline$(0,1)$ & 0 & 0 & 1 \\
\hline$(0,0)$ & 0 & 0 & 1
\end{tabular}

\begin{tabular}{c|c|c|c}
$\mathbf{x}$ & $\pi(2 \mid \mathbf{x})$ & $\pi(1 \mid \mathbf{x})$ & $\pi(0 \mid \mathbf{x})$ \\
\hline$(2,2)$ & 1 & $\max \left(\kappa_{1}^{12}, \kappa_{2}^{12}\right)$ & $\max \left(\kappa_{1}^{02}, \kappa_{2}^{02}\right)$ \\
\hline$(2,0)$ & 0 & $\kappa_{1}^{12}$ & 1 \\
\hline$(0,2)$ & 0 & $\kappa_{2}^{12}$ & 1 \\
\hline$(0,0)$ & 0 & 0 & 1
\end{tabular}

As observed in the previous section when comparing the uncertain OR to the noisy OR, the simultaneous presence of a number of causes, which, taken in isolation, do not normally produce an effect, may lead to a plausible effect under a noisy MAX, which can never be the case with an uncertain MAX. Yet situations of this kind do arise in applications and are fully compatible with possibility theory. In order to make the elicitation of possibility tables describing such situations easy, an appropriate uncertain gate has to be designed, by providing a suitable uncertain function $f$ which can trigger an effect through the accumulation of enough weak causes. One idea we have tested in order to approximate such behavior is the proposal of the uncertain MAX with thresholds, described in Section 4, which, in addition to the usual parameters of an uncertain MAX, takes a threshold $\theta_{j}$ for each value $y_{j}$ of the effect variable $Y$ (threshold gates also exist in the probabilistic setting [6]). Such threshold is an integer expressing the minimum number of causes that have to concur in order for effect $y_{j}$ to become possible.

\section{Implementation}

A prototype involving the uncertain connectives defined above, allowing to execute possibilistic models such as the one described in Section 5 has been implemented in R. Here, we give some details about the practical implementation of the uncertain connectives defined in the paper. Due to space limitations, we focus in particular on the uncertain MAX (and its variant with thresholds), whose implementation is non-trivial.

The way the uncertain MAX is implemented is shown in Algorithm 1. The parameter prm taken as input by this algorithm may be thought of as representing a set of rules of the form

$$
X_{i_{1}}=x_{i_{1}} \wedge \ldots \wedge X_{i_{m}}=x_{i_{m}} \Rightarrow Y \sim\left(\kappa\left(y_{1}\right), \ldots, \kappa\left(y_{n}\right)\right),
$$

where the $X_{i_{j}}$ on the left-hand side are parent variables of $Y$ in the possibilistic graphical model, the $x_{i_{j}}$ are one of their values, and $\left(\kappa_{1}\left(y_{1}\right), \ldots, \kappa_{1}\left(y_{n}\right)\right)$ is a normalized possibility distribution over the values of variable $Y$, i.e., for all $y \in Y, \kappa(y) \in[0,1]$, and $\max _{y \in Y} \kappa(y)=1$. Notice that this generalizes the uncertain gates to the case of multivalued variables. The left-hand side of a rule may be empty (i.e., $m=0$ ): in that case, the rule is interpreted as if it were

$$
\top \Rightarrow Y \sim\left(\kappa_{\ell}\left(y_{1}\right), \ldots, \kappa_{\ell}\left(y_{n}\right)\right) .
$$




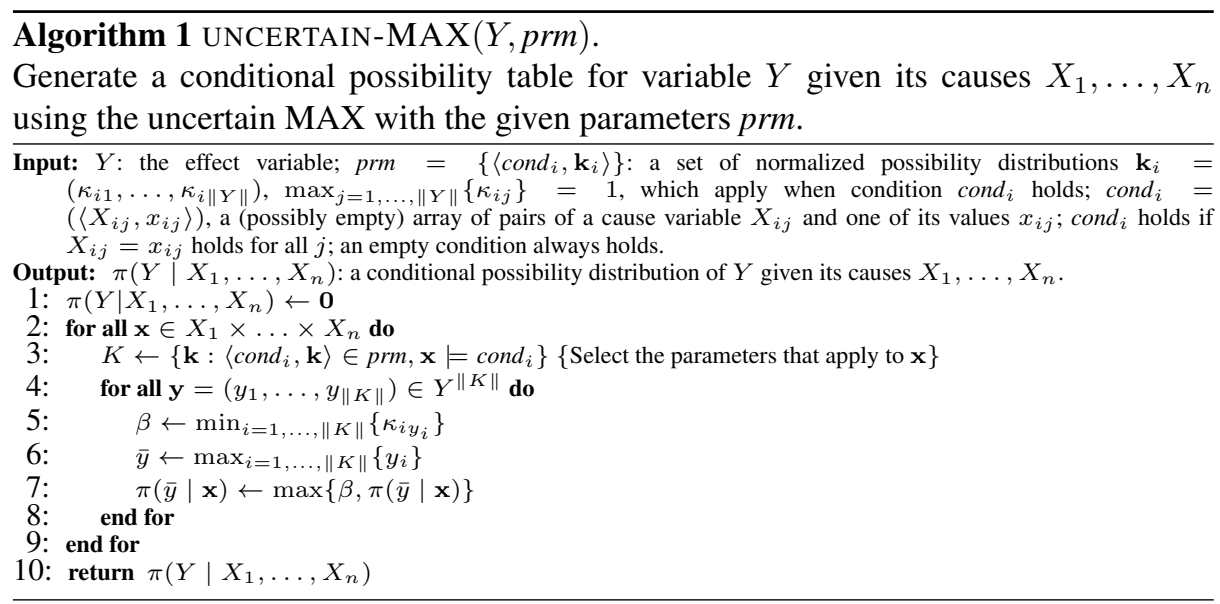

Such rules may be used to represent leak coefficients, which apply to all possible combinations of causes.

The antecedents of the rules fed into the uncertain MAX must cover all possible combinations $\mathrm{x} \in X_{1} \times \ldots \times X_{n}$ of the values of the parent variables of $Y$ in order to ensure that the resulting conditional possibility distribution $\pi\left(Y \mid X_{1}, \ldots, X_{n}\right)$ be normalized. We may notice that, if a leak rule of the form of Eq. 8 is given, that rule alone already covers all combinations of parent variable values and is thus a sufficient condition for the normalization of $\pi\left(Y \mid X_{1}, \ldots, X_{n}\right)$; in that case, the parameters of the uncertain MAX may be underspecified.

The uncertain MAX with thresholds, whose implementation is shown in Algorithm 2, has an additional parameter, which consists of an array of thresholds $\left(\theta_{1}, \ldots, \theta_{\|Y\|}\right)$, with $\theta_{i} \in\left\{1,2, \ldots,\left\|X_{1} \times \ldots \times X_{n}\right\|\right\}$. Each threshold is associated with one value $y$ of $Y$ and represents the minimal number of combinations of the causes for which $y$ is more possible than the baseline possibility given by the leak coefficients $\left(\kappa(y)>\kappa_{\ell}(y)\right)$, or zero if no leak is provided.

\section{Application}

The metropolitan area of Aix-Marseille in southern France has experienced ongoing social polarization since the 1980s. The geography of unemployment, on the one hand, and the concentration of high-skilled professionals, on the other, contribute considerably to the structuring of a contrasted metropolitan social morphology [5, 9]. Knowledge of factors inducing social polarization of the municipalities in the metropolitan area is nevertheless uncertain. Several factors contribute to the valorization or to the devalorization of the municipal residential space. But these factors have "soft", uncertain impacts on the phenomena under investigation: the same causes can not always lead to the same effects. A probabilistic model of these socio-spatial mechanisms has already been proposed [15] (cf. Fig. 1) in the form of a Bayesian network (BN). The $\mathrm{BN}$ was built using expert knowledge elicited through noisy logical gates (OR, AND, 


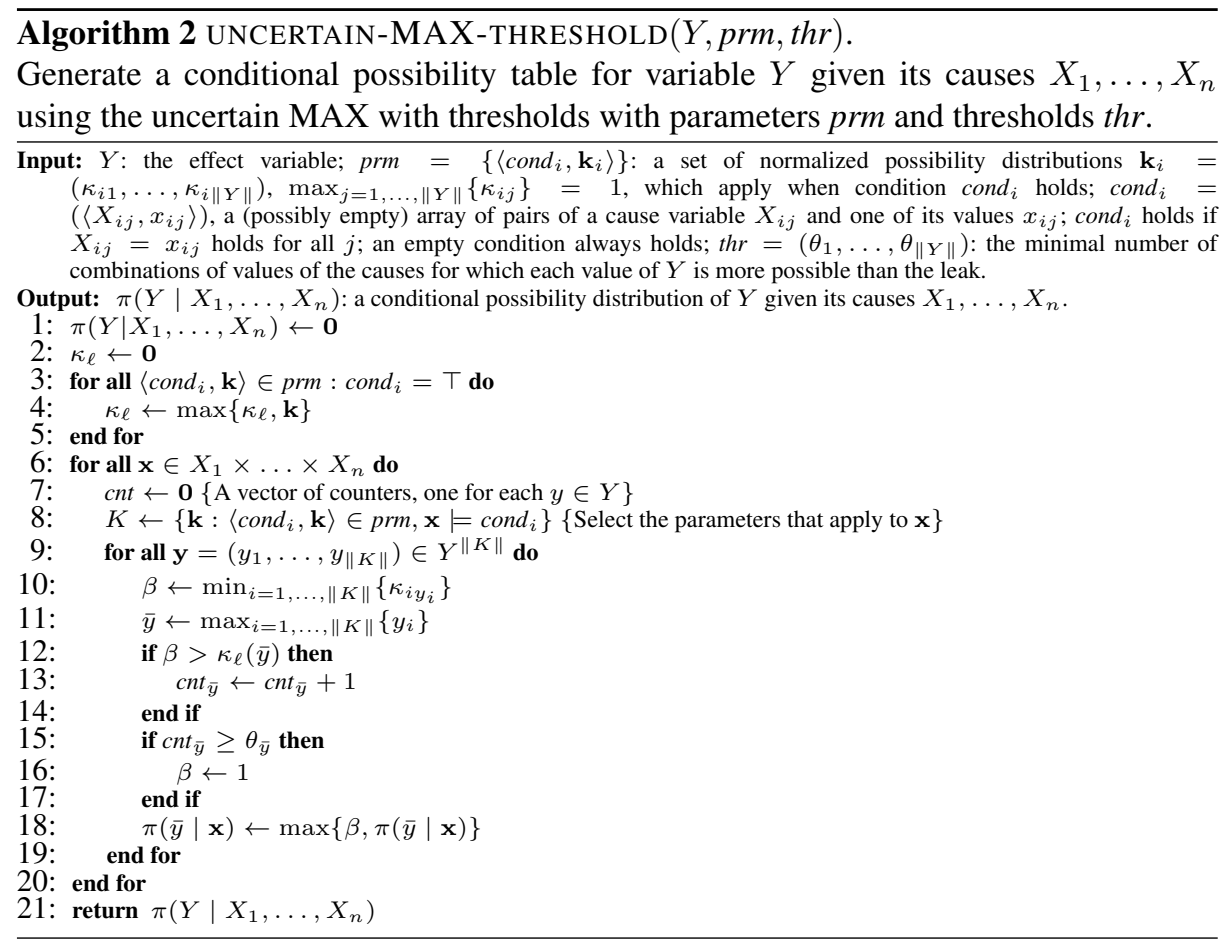

and MAX). We thus developed a possibilistic network (PN) using uncertain logical gates (OR, AND, standard MAX, and MAX-threshold) in order to link the same 26 variables of the $\mathrm{BN}$ (there is only one (ternary) MAX-threshold, with 7 parents). The parametrization of the $\mathrm{PN}$ was made compatible with the $\mathrm{BN}$ parametrization using the "most prudent" probability-to-possibility preference-preserving transformation, i.e., the $T_{1}^{-1}$ converse transformation of [8], well suited to treating subjective probabilities, in order to transform probabilistic parameters into possibilistic ones.

In Fig. 2 we show how an Uncertain OR logical gate can be used to generate a TPC. Only three parameters must be elicited: the possibilistic force of the two parent variables on the child variable (necessity of the consequence given that the parents are sufficient causes) and the leak parameter, which takes into account the activation of the consequence from secondary causes not included in the model. This table allows possibilistic inference from uncertain knowledge. If, for example, for a given municipality of the study area, we are relatively certain of having natural areas $(\Pi=1, N=0.5)$ and if it is only partially possible that we have valorized agricultural areas $(\Pi=0.5)$, we can infer that it is relatively certain $(N=0.5)$ that the municipality in question has environmental amenities.

Another difference with the probabilistic model is the possibility of keeping track of the $k_{i}$ parameters in the inference, in order to follow the sensitivity of results to the parameters of uncertain causation. The advantage of uncertain logical gates can be better appreciated in the whole model (Fig. 1). Evolution is, for example, a ternary 


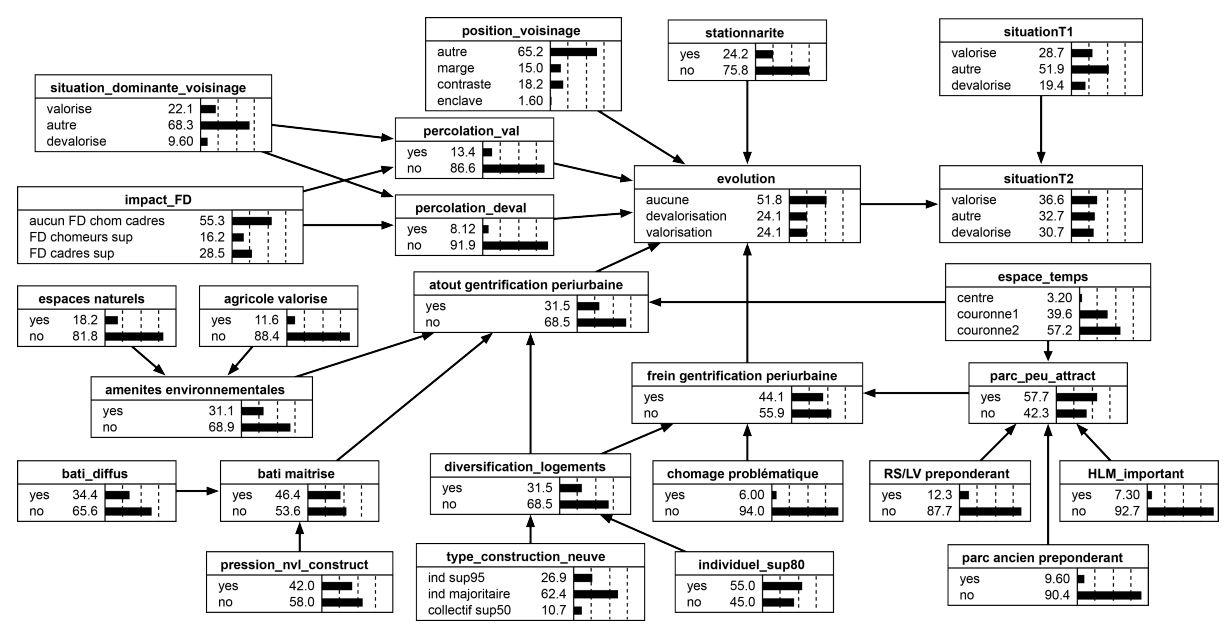

Fig. 1. The BN model for the valorization/devalorization of municipalities in the study area (from [15]).

variable (having three values: no evolution, valorization, and devalorization) depending on 5 binary variables and a 4-value variable. The TPC is thus made of $3 \times 2^{5} \times 4=384$ parameters, whereas the uncertain MAX-threshold gate used in our PN model only requires 27 parameters.

Both the BN and the PN model were thus used to produce trend scenarios for social polarization in the 439 municipalities of the Aix-Marseille metropolitan area. The future state variable that is inferred in these scenarios is the ternary variable Situation T2, having three possible values: Valorized $(V)$, Devalorized $(D)$ or Other $(O)$.

Both scenarios are based on uncertain knowledge of relationships among variables and produce uncertain evaluation of the future state of the metropolitan area in terms of social polarization. Nevertheless, the probabilistic model infers a most probable value of Situation $T 2$ for each municipality. This often gives a fallacious impression of certainty: probability differences between inferred values can be relatively small. The possibilistic model, using a min-max logic, produces in many cases sets of completely possible values $(\Pi=1)$. We thus decided to test the significance of the probability differences in the BN model: only probability differences exceeding a given threshold were considered different. For a given threshold, we could thus infer even with the BN small sets of most probable values for some municipalities.

If no threshold is considered, the most probable values inferred by the $\mathrm{BN}$ and the completely possible values inferred by the PN coincide only in $54.7 \%$ of cases. In the remaining cases, possibilistic results are more uncertain and always include probabilistic results (most probable values are always completely possible for the PN).

The best agreement between the two models is obtained with thresholds 0.20 and 0.25 (lower and higher values give worse results). $72.4 \%$ and $77.2 \%$ of the inferred values are then identical. Most probable values are almost always compatible with PN solutions: they are included in the completely possible values as, for example, when $\{V, O\}$ are the most probable values and $\{V, O, D\}$ is the set of completely possible 


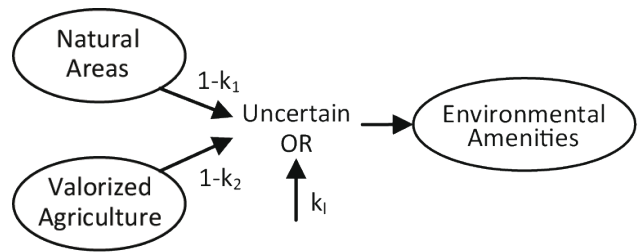

Where $\left(1-k_{i}\right)=\mathrm{N}\left(\mathrm{zi}_{\mathrm{i}} \mathrm{xi}\right)$ can be interpreted as «possibilistic force» of cause $\mathrm{xi}$ $\Pi$ (Environmental Amenities | Natural Areas, Valorized Agriculture) $=$ Uncertain OR (leak 0.1, Natural_Areas 0.8,Valorized_Agriculture 0.9)

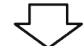

Conditional Possibility Table for variable Environmental_amenities

\begin{tabular}{|l|l|c|c|}
\hline Natural_Areas & Val_Agriculture & yes & no \\
\hline yes & yes & 1 & $\min \left(\mathrm{k}_{1}, \mathrm{k}_{2}\right)=0.1$ \\
\hline yes & no & 1 & $\mathrm{~K}_{1}=0.1$ \\
\hline no & yes & 1 & $\mathrm{~K}_{2}=0.2$ \\
\hline no & no & $\mathrm{K}_{1}=0.1$ & 1 \\
\hline
\end{tabular}

Fig. 2. Generation of a TPC through an Uncertain OR logical gate.

values. The inverse is not always the case: depending on threshold value, $24 \%$ and $18 \%$ of possibilistic solutions are not included in the most probable values.

In conclusion, uncertain logical gates made the construction of the PN model possible. The use of most probable solutions of the BN model often gives a false impression of certainty. In order to compare results from the BN and the PN models, we need to enlarge the notion of most probable values: solutions whose probabilities differ less than $0.20 / 0.25$ must be considered as equally probable. In this case, the solutions of the two models are identical for around three quarters of the municipalities of the study area. Despite this, the possibilistic model integrates a larger amount of uncertainty in the solutions inferred. Indeed, in the remaining quarter of municipalities, completely possible values inferred by the PN are normally larger sets than most probable values inferred by the $\mathrm{BN}$. The $\mathrm{BN}$ model also tends to overestimate the valorization of municipalities in the study area: the PN model often infers complete uncertainty $(\{V, O, D\}$ all equally possible) whereas the most possible values are just $V$ or $\{V, O\}$. A further analysis of the parametrization of the two models is nevertheless necessary in order to assess the origin of such a bias.

\section{Conclusion}

This is the first detailed study of the counterpart of the main probabilistic noisy gates for possibilistic networks, together with an illustrative implementation on a human geography application. Uncertain possibilistic gates are of primary interest for the practical use of possibilistic networks, when uncertainty has an epistemic flavor. The study has revealed some noticeable differences of behavior between noisy gates and uncertain possibilistic gates, in particular when the cumulation of causes having a rare effect may increase the plausibility of the effect. Generally speaking, possibilistic modeling appears to be more cautious. A detailed comparative study of the expressive power of 
Bayesian nets and possibilistic networks is a topic for further investigation, as well as the development of a complete panoply of uncertain possibilistic gates.

\section{Acknowledgments}

This work has been partially funded by CNRS PEPS Project Geo-Incertitude.

\section{References}

1. A. Antonucci. The imprecise noisy-OR gate. Proc. 14th Int. Conf. on Information Fusion (FUSION'11), Chicago, Il., July 5-8, 1-7, 2011.

2. N. Ben Amor, S. Benferhat, K. Mellouli. Anytime propagation algorithm for min-based possibilistic graphs. Soft Computing, 8, 150-161, 2003.

3. S. Benferhat, D. Dubois, L. Garcia, H. Prade. On the transformation between possibilistic logic bases and possibilistic causal networks. Int. J. Approx. Reas., 29(2), 135-173, 2002.

4. M. Caglioni, D. Dubois, G. Fusco, D. Moreno, H. Prade, F. Scarella, A. Tettamanzi. Mise en œuvre pratique de réseaux possibilistes pour modéliser la spécialisation sociale dans les espaces métropolisés. LFA'14, 267-274, 2014.

5. C. Centi. Le Laboratoire Marseillais: Chemins d'Intégration Métropolitaine et de Segmentation Sociale, Paris, L'Harmattan, 1996.

6. F. Díez, M. Drudzel. Canonical Probabilistic Models for Knowledge Engineering. Technical Report CISIAD-06-01, 2007.

7. D. Dubois, H. Prade. Possibility Theory. Plenum Press, 1988.

8. D. Dubois, H. Prade, S. Sandri. On Possibility/Probability Transformations. In: R. Lowen and M. Roubens (Eds.) Fuzzy Logic, Kluwer, 103-112, 1993.

9. G. Fusco, F. Scarella, Métropolisation et ségrégation sociospatiale. Les flux des migrations résidentielles en PACA, L'Espace Géographique, 40(4), 319-336, 2011.

10. M. Henrion. Some practical issues in constructing belief networks. In: L. Kanal , T. Levitt, J. Lemmer (eds.), Uncertainty in Artificial Intelligence 3, Elsevier, 161-173, 1989.

11. F. Jensen. Bayesian Networks and Decision Graphs. Springer, New York, 2001

12. A. Piatti, A. Antonucci, M. Zaffalon, Building knowledge-based expert systems by credal networks: a tutorial. In: Baswell, A.R. (Ed.), Advances in Mathematics Research 11, Nova Science Publishers, New York, 2010.

13. S. Parsons, J. Bigham. Possibility theory and the generalized noisy OR model. Proc. I6th Int. Conf. Inform. Proces. and Mgmt. of Uncertainty (IPMU'96), Granada, 853-858, 1996.

14. S. Renooij, L. C. van der Gaag, S. Parsons. Context-specific sign-propagation in qualitative probabilistic networks. Artif. Intell. 140(1/2), 207-230, 2002.

15. F. Scarella, La ségrégation résidentielle dans l'espace-temps métropolitain: analyse spatiale et géo-prospective des dynamiques résidentielles de la métropole azuréenne, $\mathrm{PhD}$ Thesis, Université Nice Sophia Antipolis, 2014.

16. L. A. Zadeh. Fuzzy sets as a basis for a theory of possibility. Fuzzy Sets \& Syst., 1, 3-28, 1978. 